\title{
Inversión extranjera directa y empleo manufacturero. Un análisis regional con datos de panel para México, 2007-2014
}

\author{
Leobardo de Jesús Almonte* \\ María Esther Morales Fajardo** \\ Yolanda Carbajal Suárez*
}

* Universidad Autónoma del Estado de México, México
**Universidad Nacional Autónoma de México, México

Resumen

En este artículo, se analizan los efectos de la inversión extranjera directa (IED) en la generación de empleo manufacturero en las regiones de México. A partir de estimar una función de empleo con datos de panel para cada una de las regiones del país en el periodo 2007-2014, se reporta evidencia de que existe una relación muy importante entre el crecimiento del PIB y el crecimiento del empleo para el total de la manufactura y para las diferentes regiones de México. Aún con las virtudes atribuidas a la IED, no se encontró evidencia consistente de que en las regiones la acumulación de IED sea un factor que influya en la generación de empleo manufacturero.

Palabras clave: Empleo manufacturero, inversión extranjera directa, modelos con datos de panel, regiones de México.

\section{Abstract}

Direct Foreign Investment and manufacture employment. A regional analysis with panel data for Mexico, 2007-2014

In this paper we analyze the effects of foreign direct investment (FDI) on the generation of manufacturing employment in the regions of Mexico. From the estimation of an employment function with panel data for each region of the country and for the period 2007-2014, it is found that there is a very important relationship between GDP growth and employment growth for the total of the manufacturing sector and for the different regions of Mexico. Nevertheless, there was no consistent evidence to confirm that the accumulation of FDI in the regions is a factor that influences the generation of manufacturing employment.

Key words: Manufacture employment, foreign direct investment, model with data panel, Mexico's regions. 


\section{INTRODUCCIÓN}

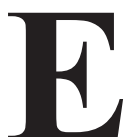

xiste un amplio consenso sobre los beneficios que aporta la inversión extranjera directa (IED) en los países de recepción, entre los cuales destacan aquellos generados en el sector productivo como la productividad y el nivel de salarios (Hanousek et al., 2011), el incremento del ahorro y la transferencia de nuevas tecnologías. Por antonomasia, los países desarrollados han sido los de mayor participación en este tipo de flujos. En 2015 las entradas de IED hacia estos países crecieron 90 por ciento, mientras que para los países en desarrollo el incremento fue sólo de 5.3 por ciento (CEPAL, 2016).

Para el caso de América Latina, aun cuando en los años recientes los flujos de IED han disminuido, la región se mantiene como una zona importante receptora de ingresos por este concepto entre el bloque de los países en desarrollo. Específicamente los flujos de IED que la región registró en 2015, como proporción de los que recibió el grueso de los países en desarrollo, representan cerca de 22 por ciento (UNCTAD, 2016). México, por su parte, ha sido uno de los principales países receptores de IED superado solamente por Brasil. En este mismo año, la IED en México fue de 30285 millones de dólares, lo que representa un incremento cercano a 18 por ciento respecto a 2014 y uno de sus niveles más altos desde 2007 (CEPAL, 2016).

Los flujos de IED cobran relevancia porque también permiten el acceso a divisas frescas, generan ingresos al gobierno y desarrollan las habilidades de la población (Dussel-Peters et al., 2003), además de que se han convertido en un factor determinante para el crecimiento económico de los países en desarrollo (Romero, 2014 y 2012; Dussel-Peters et al., 2003). Sobre todo porque, como argumentan Mejía et al. (2013), la inversión es una variable que trasciende tanto en el corto como en el largo plazo, pues tiene dos efectos importantes sobre el comportamiento presente y futuro de cualquier economía: es el segundo componente más importante de la demanda y contribuye al desarrollo económico futuro a través de la expansión del acervo de capital.

En este sentido, el papel de la IED es relevante porque, a diferencia de la inversión extranjera en cartera, permite el establecimiento de una empresa nueva y/o la adquisición o transformación de bienes inmuebles y empresas. Más aún, para las economías en desarrollo los flujos de in- 
versión son un factor necesario para alcanzar sus objetivos de crecimiento (Trevino et al., 2002). De ahí que prácticamente todos los países en desarrollo buscan de manera activa atraer montos importantes de IED. Para el caso de México, desde 1980 se ha buscado activamente atraer una mayor cantidad de flujo: primero, relajando las restricciones a la entrada y con cambios en la reglamentación de la ley de inversión extranjera; ${ }^{1}$ después, con la puesta en marcha del Tratado de Libre Comercio con América del Norte (TLCAN), que entre sus objetivos estaba la atracción de este tipo de inversión extranjera, se favoreció la entrada de flujos importantes de IED (Romero, 2014 y 2012).

Sin embargo, aún con las virtudes que se le atribuyen a la IED, en la literatura especializada los análisis se han enfocado en los determinantes de estos flujos de inversión en los mercados laborales, sobre la productividad y los salarios (Aitken and Harrison, 1999; Hanousek et al., 2011), específicamente para la manufactura, no existe evidencia suficiente que permita argumentar que ha tenido efectos importantes en la generación de empleos. Destacan los estudios de Jude y Pop (2015) que examinan el papel de la IED como determinante del empleo por medio de una estimación de panel para 20 países de Europa del Este y Central, en el periodo 19952012 para los sectores de servicios y manufactura; así como el estudio de Onaran (2008) que considera ocho países europeos, se enfoca en el sector manufacturero y emplea un modelo de panel. En ambos casos, se destaca que los efectos de la inversión en la generación de empleos son marginales.

En el caso mexicano se ha insistido que para el análisis de la IED es necesario considerar su asociación con el empleo (Dussel-Peters et al., 2003). Entre los estudios específicos relacionados con el tema, destacan los trabajos de Vergara et al. (2015), quienes analizan la relación de la IED y el empleo en el sector industrial en la región norte de México, y de Loría y Brito (2005) quienes evalúan el impacto de la IED en el empleo sectorial de México y a partir de un ejercicio de prospectiva concluyen que por la fuerte inercia de la globalización, se le puede atribuir a la IED un importante factor de crecimiento y de creación de empleos.

En este contexto, el objetivo de este artículo es analizar los efectos de la IED en la generación de empleo de la manufactura en México por regiones, a partir de estimar con datos de panel una función de empleo para cada una de las regiones del país. Además de la introducción y las conclusiones, el artículo se integra de tres apartados: en el primero se hace una revisión

${ }^{1}$ La legislación sobre la IED ha sufrido cambios importantes desde finales de los ochenta, particularmente con la nueva Ley de Inversión Extranjera de 1993 y su reglamento de 1998 y modificaciones sectoriales, tal como en el sector financiero (Dussel-Peters et al., 2003: 82). 
de la literatura disponible que da evidencia de la importancia de los estudios del tema en México; en el segundo se analiza la evolución de los flujos de IED desde finales de los años ochenta del siglo XX, y con mayor detalle para las regiones de México en el periodo 1994-2014; finalmente, en el tercer apartado, se estima una función de empleo de la manufactura para 2007-2014, para el total de la economía y para cada una de las cinco regiones que se describen con detalle en el apartado correspondiente. Se enfatiza en el papel de la IED y de la producción manufacturera como factores que explican la generación de empleos en este sector.

\section{INVERSIÓN EXTRANJERA DIRECTA Y EMPLEO EN MÉXICO. REVISIÓN DE LITERATURA}

La literatura disponible da evidencia de la importancia que en los años recientes ha cobrado el estudio de la IED. Las líneas de trabajo son diversas, para México destacan aquellos que explican los determinantes de la IED (Ángeles-Castro y Ortiz-Galindo, 2010; Mejía, 2005); los que han asociado el impacto que la inversión extranjera tiene en el crecimiento económico de México (Romero, 2012; Aguayo, 2004), en la manufactura (Mendoza, 2011), y los que han evaluado los impactos sobre el empleo (Chiatchoua et al., 2016; Vergara et al., 2015; Loría y Brito, 2005 Turner y Martínez, 2003; Gouset, 2000).

De los primeros, los resultados que se reportan ilustran el estado del arte en los estudios de la IED; sobre todo porque dejan ver que entre los factores que se asocian a los flujos de la IED están aquellos relacionados con las posibilidades de crecimiento, de ahí que se argumente que la IED tiende a fluir hacia las regiones que tienen mayor ingreso per cápita y un PIB más alto, por lo que la IED está determinada por la oferta de mano de obra calificada, la cercanía a mercados mayores, la disponibilidad de infraestructura y en generar mejores niveles de desarrollo (Ángeles-Castro y Ortiz-Galindo, 2010); y aquellos factores relacionados con el potencial del mercado, la calidad de los factores de la producción, las políticas gubernamentales y su efectividad, y la estabilidad política o laboral y la seguridad pública (Mejía, 2005). La inversión fluye hacia los lugares en los cuales hay actividades productivas importantes o donde ya existen empresas transnacionales, lo cual sugiere la importancia de las economías de aglomeración e indica cierta tendencia hacia la concentración de la IED en pocos estados del país (Mejía, 2005).

Más aún, para los inversionistas extranjeros no es importante si los trabajadores están calificados o no, si no que ellos buscan las zonas del país 
que generan menores costos de producción (Aguayo, 2004). En este sentido, los estados del norte de México han captado mayor IED principalmente por el desplazamiento de la industria manufacturera fuera de la ciudad de México en gran medida por sus ventajas de localización con respecto al mercado de Estados Unidos (Aguayo, 2004).

En otra línea de argumentación, Romero (2012) ha referido que el impacto de la IED en el crecimiento económico de México no es de magnitudes importantes y que bajo la actual estrategia, el motor de crecimiento es la inversión privada nacional más que la IED; por lo que la IED debería ser considerada sólo como complementaria, pero no central para el proceso de crecimiento. Más aún, se ha argumentado que la IED ha sido decisiva en el crecimiento de las economías receptoras, a partir de que se relaciona con el incremento del acervo de capital existente en la economía, de la mayor productividad del trabajo en las empresas multinacionales, de la generación de economías externas en dicho sector industrial, en especial en regiones donde existe una alta concentración de IED y difusión de la tecnología (Mendoza, 2011).

Con relación a los efectos sobe la manufactura, Mendoza (2011), a partir de estimar un modelo con datos en panel dinámico, demuestra que el efecto de la IED en el crecimiento de las manufacturas en México se relaciona con el efecto positivo de las exportaciones manufactureras y la expansión de la calificación de la fuerza de trabajo en el sector productivo más que por la hipótesis de la existencia de efectos positivos de la IED en el crecimiento de la industria manufacturera al nivel sub-sectorial.

Con relación a los efectos que la IED tiene en la generación de empleos, son pocos los trabajos para México; entre ellos el de Turner y Martínez (2003), quienes analizan el impacto que durante 1990 y el 2000 tuvo la IED en México. Entre los resultados a los que llegan es que la inversión extranjera ha proporcionado un apoyo fundamental al desarrollo de las empresas multinacionales, de la industria exportadora y de la industria maquiladora. Por su lado, Loría y Brito (2005) analizan el impacto de la IED en el empleo sectorial de México y a partir de un ejercicio de prospectiva concluyen que por la fuerte inercia de la globalización, se le puede atribuir a la IED un importante factor de crecimiento y generador de empleos.

Entre los trabajos recientes se han destacado los efectos positivos que la IED tiene sobre el empleo (Chiatchoua et al., 2016), particularmente en tres líneas de análisis: i) cuando la IED complementa la inversión nacional, porque supone la creación de nuevas empresas y, en consecuencia, el incremento en la demanda de trabajo; ii) en el caso de si los flujos de IED 
se concentran en las industrias intensivas en trabajo el aumento será sustancial por la posibilidad de los encadenamientos hacia atrás o hacia adelante con empresas locales; iii) por los efectos en la calidad del empleo, las empresas con capital extranjero usan una mayor proporción de empleados calificados que las empresas sin estos capitales.

Además, se empieza a priorizar en el estudio de los efectos territoriales de la IED. De la literatura disponible para México, Vergara y Carbajal (2015) analizan el comportamiento de los flujos de IED hacia las entidades federativas para determinar la posible formación de aglomeraciones espaciales entre ellas. Sus resultados apuntan que no existe un efecto contagio que favorezca la concentración de la IED total o sectorial en una zona geográfica concreta. Vergara et al., (2015) presentan evidencia para la región norte de México en el sentido de que evalúan el impacto de la IED en el empleo del sector industrial. La evidencia que reportan es que, al menos para la región norte de México, la IED no resulta ser un factor determinante para impulsar el empleo en la industria de la región norte del país, excepto para la división cinco que comprende la industria eléctrica, cuya característica es que es una industria intensiva en mano de obra.

En otra línea de trabajo se discute que la dinámica de crecimiento de la IED se asocia a un sólido proceso de divergencia regional (Torres et al., 2017), en el que la capacidad de las entidades federativas para atraer IED depende más en términos de infraestructura y de estrategias comerciales para los inversionistas que de las virtudes de las cadenas productivas que se pueden generar con los estados vecinos. En ese sentido, los autores argumentan que la IED debe acompañarse por una visión regional que promueva los incentivos y condiciones para motivar la localización de la inversión extranjera en regiones con limitaciones para capturarla. Sobre el mismo argumento, Aguayo (2004) atribuye que la IED explica, en parte, la divergencia regional de México, a partir de que la eliminación de las restricciones al comercio internacional y los controles a la inversión extranjera directa, junto con el auge económico de Estados Unidos, incrementaron en gran medida las exportaciones y los flujos de IED, particularmente a los estados más ricos, que se localizan cerca de la frontera norte.

INVERSIÓN EXTRANJERA DIRECTA Y EMPLEO EN LA MANUFACTURA en las Regiones de MÉXICO

La inversión extranjera directa en el contexto nacional 
Como se ha comentado, aun cuando los flujos de IED hacia la región de América Latina han disminuido en los años recientes, se mantiene como una importante receptora de IED entre el bloque de los países en desarro1lo. Específicamente, las principales economías de América Latina se han convertido en las grandes receptoras de IED, sobre todo a partir de los años noventa del siglo XX. En este caso, Brasil durante varios años se convirtió en el principal receptor, seguido de México y Argentina (Figura 1). Sin embargo, si analizamos los flujos de IED como proporción del PIB, destaca el caso de la economía chilena, cuya contribución en los años recientes es de alrededor de ocho por ciento, mientras para las economías grandes de América Latina (Argentina, Brasil y México) fluctúa entre dos y cuatro por ciento (Figura 2), sobre todo desde los primeros años del siglo XXI.

La Comisión Económica para América Latina (CEPAL) ha reportado que esta tendencia ha beneficiado principalmente al sector de los servicios, seguido por las manufacturas; lo que deja ver un patrón de especialización productiva en el que los flujos de inversión están incentivados por la búsqueda de eficiencia en los sectores de servicios y de la manufactura, seguida por la búsqueda de materias primas (CEPAL, 2013). Desafortunadamente este patrón de especialización productiva ha seguido básicamente la mano de obra barata o poco especializada y las materias primas que ofrece la región (Morales et al., 2015).

Para el caso de México, es evidente que el inicio del Tratado de Libre Comercio con América del Norte favoreció el incremento en los flujos de IED, tal como se puede ver en la Figura 3; sin embargo, aun cuando la IED ha crecido de manera importante en estos años y ha impactado a algunos sectores, no es claro el efecto que ha tenido en el fortalecimiento al crecimiento de largo plazo ni en la generación de empleos.

Mejía (2005) ha sugerido que la dinámica de la IED ha estado estrechamente vinculada al comportamiento del crecimiento económico y que las entradas de capital aumentan en los periodos de expansión y disminuyen en los periodos de estancamiento y aun cuando los flujos de IED en los años recientes han crecido de manera importante, sobre todo desde finales de los años ochenta del siglo XX; sin embargo, como el mismo autor argumenta, los efectos de la IED han sido desiguales en el sentido de que las empresas orientadas hacia la exportación - incluyendo maquiladoras y 


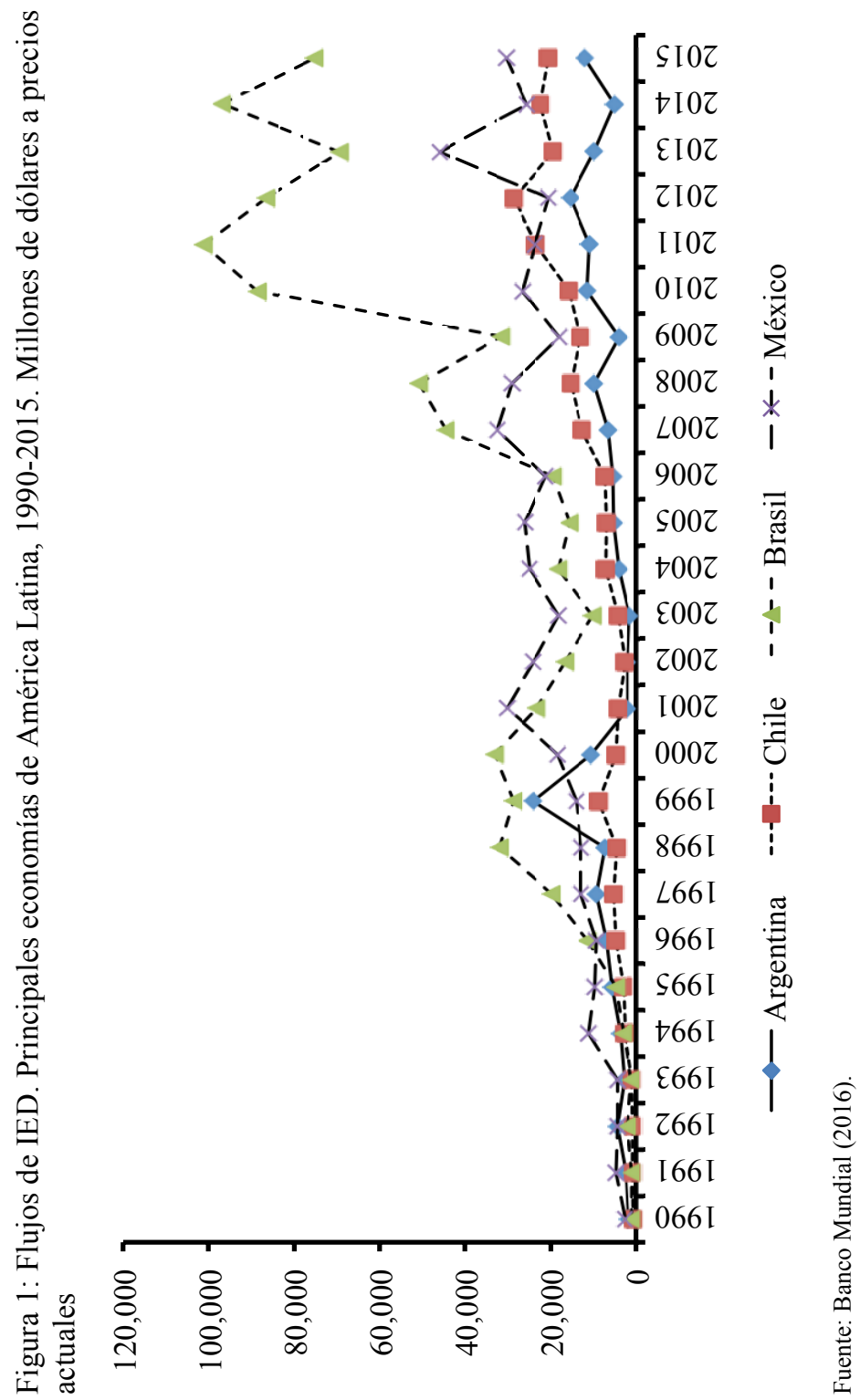


Inversión extranjera directa y empleo manufacturero. Un análisis regional con datos ... / L. de J. ALMONTE et al.

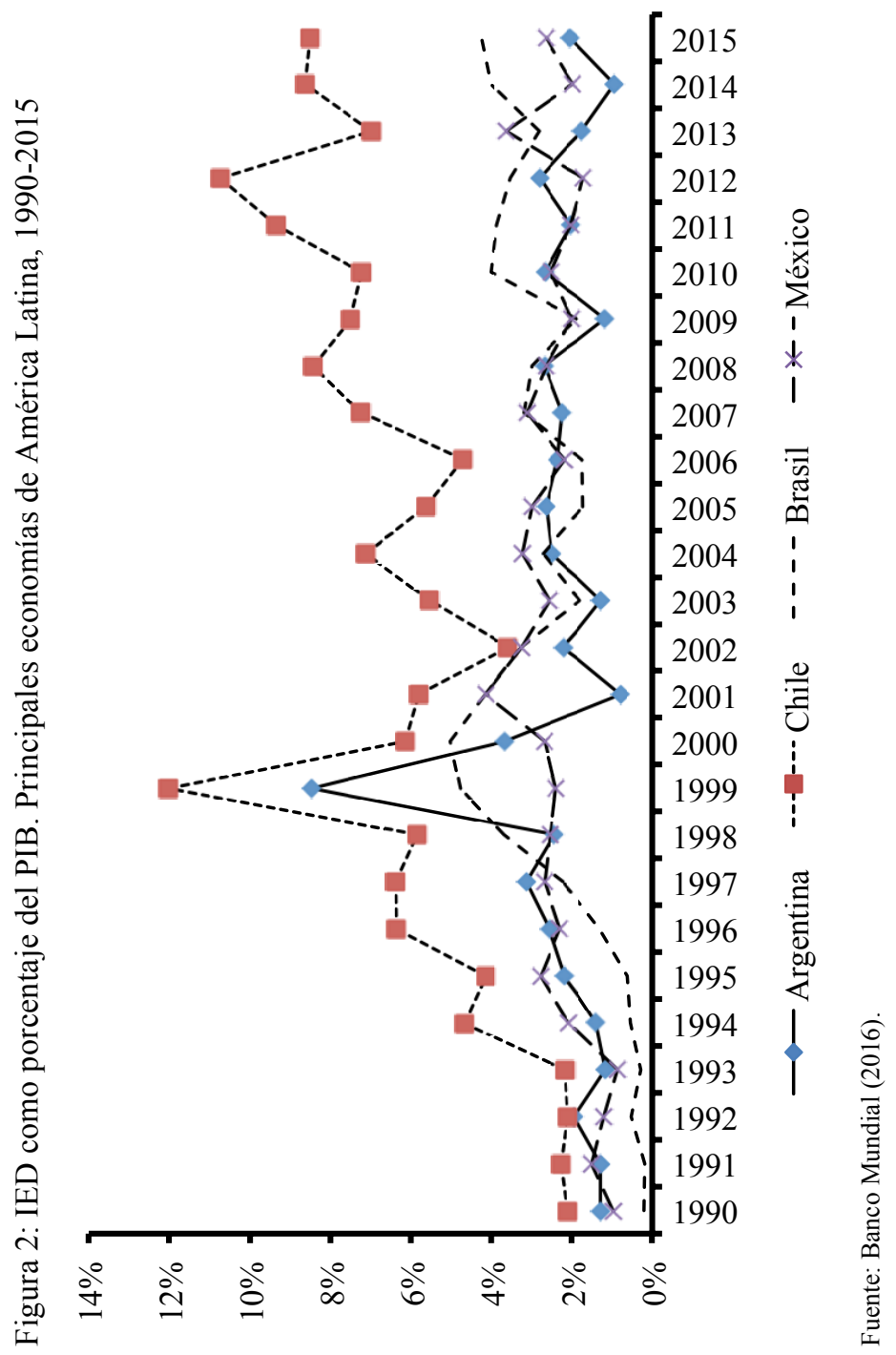


exportadoras indirectas - se han beneficiado enormemente con las reformas económicas.

Efectivamente, a lo largo de los últimos veinticinco años los flujos de IED en México han experimentado un crecimiento muy importante, tal como se aprecia en la Figura 3, con efectos importantes en algunos sectores. Díaz et al. (2011) documentan que la industria manufacturera, de manera tradicional, ha liderado la captación de capitales provenientes del exterior y que otros sectores, como el financiero y el comercial, también tienen una participación significativa para el destino de la IED. Al respecto, Mendoza (2011) argumenta que la entrada de IED al país en los años noventa del siglo XX tuvo un gran impacto en la economía mexicana, principalmente la IED destinada al sector manufacturero. Pero este sector ha ido perdiendo fuerza en gran medida por el papel que ha jugado el sector servicios y por las diferentes crisis que ha experimentado Estados Unidos, principal socio comercial de México.

Como se observa, es evidente la dinámica de los flujos de IED en México y la necesidad de revisar lo que ha ocurrido a nivel de entidades federativas y de regiones en el país, sobre todo porque este proceso global del que se acompañan los flujos de IED en el mundo afecta de manera específica a sectores productivos y regiones, de ahí que es importante examinar la forma en que se ha dado la distribución regional/estatal de la IED en México.

\section{Inversión extranjera directa y empleo en las regiones de México}

Para el análisis, el país se dividió en cinco regiones. Esta división se plantea en un sentido estrictamente funcional que busca identificar regularidades en entidades o regiones específicas, la propuesta de regionalización con la que se trabaja agrupa a las entidades de acuerdo con el desarrollo de su actividad manufacturera y por su relación con el sector externo. Se ubica en una región a todos los estados fronterizos (Frontera Norte) para destacar el auge —en el crecimiento de la actividad manufacturera, recepción de IED y, sobre todo, en la generación de empleos- que ha tenido en los últimos años como consecuencia de su relación fronteriza con el mercado más grande, Estados Unidos. En otra región a los estados del Norte, que no son fronterizos (región Norte); a la Centro-Este porque es importante destacar el auge manufacturero de Guanajuato y de Jalisco; la fuerza de la región Centro Oeste con el Distrito Federal, Estado de México, y el crecimiento, relativamente reciente, de las actividades manufactureras de Puebla y Querétaro y; la región Sur, cuya vocación industrial es limitada. De ahí que se 
Inversión extranjera directa y empleo manufacturero. Un análisis regional con datos ... / L. de J. ALMONTE et al.

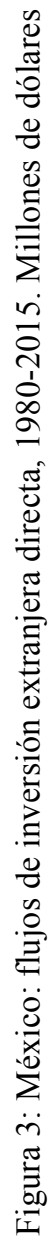

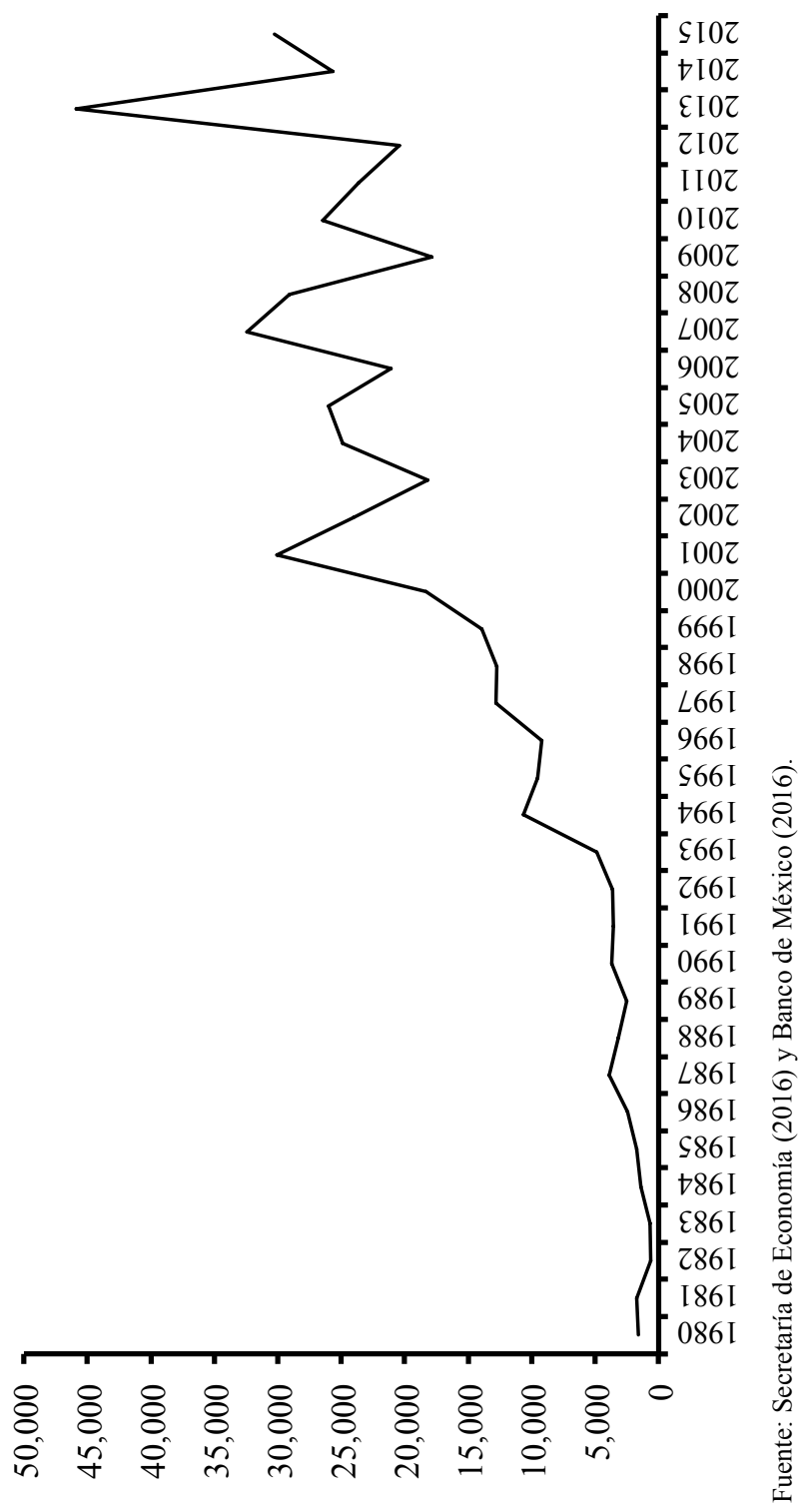


Tabla 1: México: inversión extranjera directa, 1994-2014 (millones de dólares)

\begin{tabular}{|c|c|c|c|c|c|c|c|c|c|c|}
\hline \multirow[t]{2}{*}{ Región } & & \multicolumn{3}{|c|}{ Promedio anual del periodo } & \multicolumn{3}{|c|}{$\%$ de la IED total } & \multicolumn{3}{|c|}{$\begin{array}{l}\% \text { de la IED total de la } \\
\text { región }\end{array}$} \\
\hline & & $\begin{array}{l}1994 \\
2007 \\
\end{array}$ & $\begin{array}{l}2008 \\
2009 \\
\end{array}$ & $\begin{array}{l}2008 \\
2014 \\
\end{array}$ & $\begin{array}{l}1994 \\
2007 \\
\end{array}$ & $\begin{array}{l}2008 \\
2009 \\
\end{array}$ & $\begin{array}{l}2008 \\
2014 \\
\end{array}$ & $\begin{array}{l}1994 \\
2007 \\
\end{array}$ & $\begin{array}{l}2008 \\
2009 \\
\end{array}$ & $\begin{array}{l}2008 \\
2014 \\
\end{array}$ \\
\hline Nacional & & $18,251.8$ & $23,108.8$ & $25,929.1$ & 100.0 & 100.0 & 100.0 & & & \\
\hline \multirow{7}{*}{ 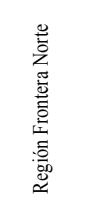 } & Baja California & 815.1 & 921.0 & 854.3 & 4.5 & 4.0 & 3.3 & 18.7 & 16.6 & 16.2 \\
\hline & Coahuila & 179.9 & 637.4 & 533.8 & 1.0 & 2.8 & 2.1 & 4.1 & 11.5 & 10.1 \\
\hline & Chihuahua & 845.1 & $1,307.0$ & $1,338.2$ & 4.6 & 5.7 & 5.2 & 19.4 & 23.6 & 25.4 \\
\hline & Nuevo León & $1,899.2$ & $1,550.2$ & $1,783.4$ & 10.4 & 6.7 & 6.9 & 43.6 & 28.0 & 33.8 \\
\hline & Sonora & 230.8 & 781.4 & 338.5 & 1.3 & 3.4 & 1.3 & 5.3 & 14.1 & 6.4 \\
\hline & Tamaulipas & 388.4 & 346.7 & 420.7 & 2.1 & 1.5 & 1.6 & 8.9 & 6.3 & 8.0 \\
\hline & Total & $4,358.5$ & $5,543.7$ & $5,269.0$ & 23.9 & 24.0 & 20.3 & 100.0 & 100.0 & 100.0 \\
\hline \multirow{7}{*}{ 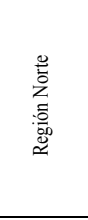 } & Aguascalientes & 87.9 & 398.8 & 386.7 & 0.5 & 1.7 & 1.5 & 15.3 & 20.4 & 17.1 \\
\hline & Baja California Sur & 200.2 & 336.7 & 369.7 & 1.1 & 1.5 & 1.4 & 34.8 & 17.3 & 16.3 \\
\hline & Durango & 72.2 & 317.6 & 545.7 & 0.4 & 1.4 & 2.1 & 12.5 & 16.3 & 24.1 \\
\hline & San Luis Potosí & 40.1 & 35.0 & 139.9 & 0.2 & 0.2 & 0.5 & 7.0 & 1.8 & 6.2 \\
\hline & Sinaloa & 109.8 & 65.4 & 238.2 & 0.6 & 0.3 & 0.9 & 19.1 & 3.4 & 10.5 \\
\hline & Zacatecas & 66.0 & 797.2 & 585.6 & 0.4 & 3.4 & 2.3 & 11.5 & 40.9 & 25.8 \\
\hline & Total & 576.1 & $1,950.8$ & $2,265.9$ & 3.2 & 8.4 & 8.7 & 100.0 & 100.0 & 100.0 \\
\hline \multirow{6}{*}{ 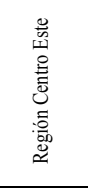 } & Colima & 16.3 & 17.3 & 30.1 & 0.1 & 0.1 & 0.1 & 2.0 & 1.9 & 1.7 \\
\hline & Guanajuato & 111.4 & 212.2 & 438.0 & 0.6 & 0.9 & 1.7 & 14.0 & 23.8 & 24.2 \\
\hline & Jalisco & 494.8 & 589.7 & 982.6 & 2.7 & 2.6 & 3.8 & 62.2 & 66.1 & 54.3 \\
\hline & Michoacán & 117.8 & 30.2 & 263.2 & 0.6 & 0.1 & 1.0 & 14.8 & 3.4 & 14.5 \\
\hline & Nayarit & 55.7 & 43.0 & 96.6 & 0.3 & 0.2 & 0.4 & 7.0 & 4.8 & 5.3 \\
\hline & Total & 795.9 & 892.3 & $1,810.5$ & 4.4 & 3.9 & 7.0 & 100.0 & 100.0 & 100.0 \\
\hline \multirow{8}{*}{ 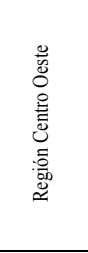 } & Distrito Federal & $10,615.9$ & $11,954.2$ & $13,399.4$ & 58.2 & 51.7 & 51.7 & 87.1 & 83.4 & 84.1 \\
\hline & Hidalgo & 14.9 & 20.3 & -37.7 & 0.1 & 0.1 & -0.1 & 0.1 & 0.1 & -0.2 \\
\hline & México & 968.3 & $1,639.6$ & $1,380.3$ & 5.3 & 7.1 & 5.3 & 7.9 & 11.4 & 8.7 \\
\hline & Morelos & 111.9 & 38.9 & 45.2 & 0.6 & 0.2 & 0.2 & 0.9 & 0.3 & 0.3 \\
\hline & Puebla & 308.4 & 186.0 & 565.0 & 1.7 & 0.8 & 2.2 & 2.5 & 1.3 & 3.5 \\
\hline & Querétaro & 144.6 & 471.0 & 526.4 & 0.8 & 2.0 & 2.0 & 1.2 & 3.3 & 3.3 \\
\hline & Tlaxcala & 25.1 & 21.1 & 52.2 & 0.1 & 0.1 & 0.2 & 0.2 & 0.1 & 0.3 \\
\hline & Total & $12,189.1$ & $14,331.2$ & $15,930.8$ & 66.8 & 62.0 & 61.4 & 100.0 & 100.0 & 100.0 \\
\hline \multirow{9}{*}{ 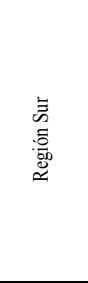 } & Campeche & 7.6 & -38.5 & -25.3 & 0.0 & -0.2 & -0.1 & 2.3 & -9.8 & -3.9 \\
\hline & Chiapas & 6.0 & 8.9 & 10.1 & 0.0 & 0.0 & 0.0 & 1.8 & 2.3 & 1.6 \\
\hline & Guerrero & 19.0 & 9.3 & 97.6 & 0.1 & 0.0 & 0.4 & 5.7 & 2.4 & 15.0 \\
\hline & Oaxaca & 3.4 & 24.0 & 65.7 & 0.0 & 0.1 & 0.3 & 1.0 & 6.1 & 10.1 \\
\hline & Quintana Roo & 166.9 & 177.5 & 263.4 & 0.9 & 0.8 & 1.0 & 50.2 & 45.4 & 40.3 \\
\hline & Tabasco & 33.9 & 27.9 & 69.1 & 0.2 & 0.1 & 0.3 & 10.2 & 7.1 & 10.6 \\
\hline & Veracruz & 55.7 & 157.8 & 138.4 & 0.3 & 0.7 & 0.5 & 16.8 & 40.4 & 21.2 \\
\hline & Yucatán & 39.6 & 24.0 & 33.9 & 0.2 & 0.1 & 0.1 & 11.9 & 6.1 & 5.2 \\
\hline & Total & 332.1 & 390.9 & 652.9 & 1.8 & 1.7 & 2.5 & 100.0 & 100.0 & 100.0 \\
\hline
\end{tabular}

agruparon las entidades federativas del país en cinco regiones, de acuerdo como se presentan en la Tabla 1.

Si se revisan los datos de IED por entidad federativa de destino para los años recientes que corresponden al proceso de apertura comercial con el inicio de las operaciones del Tratado de Libre Comercio de América del Norte; específicamente, si se divide el periodo 1994-2014 en dos (19942007 y 2008-2014, con la idea de observar posibles cambios resultado de la Gran Recesión de 2008-2009), se pueden identificar algunos hechos im- 
portantes de lo que ocurre en las regiones. En principio - sin considerar al Distrito Federal, ahora Ciudad de México, que en promedio concentró 58.2 y 51.7 por ciento del total de los flujos de IED que recibió el país en los periodos 1994-2007 y 2008-2014-, las entidades que se han convertido en las receptoras de IED más importantes después de la Ciudad de México son Nuevo León, Estado de México, Chihuahua, Baja California y Jalisco. Sin embargo, en el caso de Nuevo León se registró una disminución importante en el volumen de los flujos de IED (de 10.4 por ciento en el periodo 1994-2007 a 6.9 por ciento en el periodo 2008-2014), lo mismo que Baja California ( 4.5 y 3.3 por ciento, para los mismos periodos); mientras que el Estado de México se mantuvo en 5.3 por ciento, y Chihuahua y Jalisco registraron crecimiento (de 4.6 a 5.2 por ciento y de 2.7 a 3.8 por ciento, respectivamente para los mismos periodos) (Tabla 1).

$\mathrm{Al}$ analizar por regiones, se identificó una dinámica diferente (Tabla 1). Fue claro que la región Centro Oeste, que incluye al Distrito Federal, concentró parte importante de los flujos de IED que llegaron a México (66.8 y 61.4 por ciento para los periodos 1994-2007 y 2008-2014), le siguió la Frontera Norte con poco más de 20 por ciento, la región Norte, la Centro Este y, finalmente, la Sur, cuya captación de IED fue muy baja (2.5 por ciento del total nacional para el periodo 2008-2014).

En este contexto, si se tratara de identificar los efectos que la recesión de 2008-2009 generó en la captación de IED en las regiones, se observa que a excepción de la Frontera Norte y de la región Centro Oeste, que presentaron ligeras disminuciones en el porcentaje de captación de IED respecto al total nacional, el resto de las regiones registraron aumentos: la Norte aumentó de 3.2 a 8.7 por ciento en los periodos 1994-2007 y 20082009, la Centro Este de 4.4. a siete por ciento y la región Sur de 1.8 a 2.5 por ciento, en los mismos periodos (Tabla 1).

De manera particular vale analizar con más detalle lo que ocurre con cada una de las regiones (Tabla 1). En el caso de la Frontera Norte, que después de la Centro Oeste es la que más flujos de IED recibió, Coahuila y Chihuahua fueron las entidades federativas cuya concentración de los flujos de IED fueron en ascenso: Coahuila, de 4.1 a 10.1 por ciento para los periodos 1994-2007 y 2008-2014; Chihuahua, de 19.4 a 25.4 por ciento en los mismos periodos. Destaca el caso de Nuevo León, que ha sido una de las entidades más dinámicas y de las más importantes en términos de destino de los flujos de IED, pero que entre estos periodos ha disminuido su participación en el total de los flujos de IED de la región: de 43.6 por ciento en el periodo 1994-2007 a 33.8 por ciento en el periodo 2008-2014. 
Para la región Norte, en los años recientes Zacatecas y Durango concentraron cerca del 46 por ciento de los flujos de IED a la región; sobre todo, fueron los estados en los que los flujos de IED con relación al total de la región han crecido de manera importante (para el caso de Zacatecas, en promedio la proporción de la IED recibida, con relación al total de la región, aumentó de 11.5 a 25.8 por ciento entre 1994-2007 y 2008-2014), contrario a lo que ha ocurrido con Baja California Sur y Sinaloa, cuya participación en la captación de IED ha disminuido en los años de la era del TLCAN (de 34.8 a 16.3 por ciento para Baja California Sur y de 19.1 a 10.5 por ciento para Sinaloa, para los mismos periodos). En la región Centro Este sobresalieron Jalisco y Guanajuato: del primero vale recordar que es una de las entidades cuya vocación industrial se remonta a los inicios de la industrialización en México, de ahí que esta condición sea una de las causas que expliquen que parte importante de los flujos de IED que llegan a la región se concentren en esta entidad (en el periodo 2008-2014 en promedio concentró 54.3 por ciento del total de IED de la región). En Guanajuato prácticamente se duplicó la recepción de IED entre los dos periodos, de 14 por ciento entre 1994 y 2007 a 24.2 por ciento para el periodo 2008-2014. Esta entidad es una de las más dinámicas de los años recientes producto del impulso que está teniendo el sector automotriz y de los flujos de IED que están llegando para el sector. La presencia de armadoras como General Motors y Volkswagen, en Silao, Guanajuato, ha generado una importante dinámica regional, con un incremento sustancial de productoras de partes tanto de primer nivel, como de segundo y tercero. Sin duda, esta región podría continuar en ascenso por el efecto de la instalación de dos nuevas plantas armadoras en Guanajuato: Mazda, con una inversión de 500 millones de dólares, en Salamanca, y Honda, con 800 millones de dólares invertidos en una planta de ensamble en Celaya (véase Carbajal et al., 2016).

El caso de la región Centro Oeste es destacable porque concentró poco más de 60 por ciento del total de los flujos de IED que llegaron al país. Sin embargo, más que analizar la situación particular del Distrito Federal —que en el periodo 2008-2014 en promedió registró 51.7 por ciento del total de IED del país y que se explica porque varias empresas de diferentes partes del país tienen su razón social en esta ciudad-, lo que resalta fueron los casos de Puebla y Querétaro que en promedio para los mismos años concentraron 3.5 y 3.3 por ciento del total de la IED que llegó a la región (una concentración mayor que la registrada en el periodo 1994-2007). Pareciera poco; sin embargo, los volúmenes fueron mayores que los regis- 
trados por Guanajuato y Aguascalientes que en los años recientes se han convertido en entidades importantes por la recepción de IED. El Estado de México, por su vocación industrial de muchos años, continuó como un destino importante de los flujos de IED (en el periodo 2008-2014 concentró en promedio 8.7 por ciento de la IED que registró la región (Tabla 1).

Finalmente, la región Sur. Fue la menos favorecida como destino de la IED, para el periodo 2008-2014 recibió sólo 2.5 por ciento del total de la IED que llegó al país. De las seis entidades que la integran, sólo Quintana Roo y Veracruz se identificaron como los estados más atractivos para la IED, el primero se explica por su vocación turística y el segundo por el desarrollo de la industria química, que en los años recientes ha recibido en promedio, de 2008 a 2016, 325.97 millones de dólares (Secretaría de Economía, 2017).

De esta descripción podemos observar que aparentemente existe una relación muy importante entre las condiciones socioeconómicas que caracterizan a las entidades y a las regiones del país y el destino de la IED (Dussel Peters et al., 2003 y Mejía, 2005), y que de manera más puntual algunos autores han argumentado que existe una clara relación positiva entre las entidades donde se concentra la IED y que cuentan con mayores niveles de bienestar, como los estados de Nuevo León, Baja California, Chihuahua, Estado de México, Jalisco, por ejemplo (González y Hernández, 2008).

En estas condiciones, una mayor proporción de IED en la región se reflejaría en aspectos como producción, empleo e innovación territorial (González y Hernández, 2008).

\section{INVERSIÓN EXTRANJERA DIRECTA Y EMPLEO EN LAS REGIONES DE MÉXICO. Un MOdelo CON DATOS DE PANEL}

\section{Los datos}

Se trabajó con información estadística oficial del Instituto Nacional de Estadística y Geografía (INEGI), de la Secretaría del Trabajo y Previsión Social (STyPS) y de la Secretaría de Economía (SE). Son datos anuales de cada una de las 32 entidades federativas de México para el periodo 2007-2014; en específico para las siguientes variables: i) empleo manufacturero por entidad federativa, que corresponde al personal ocupado total de la industria manufacturera de la Encuesta mensual de la industria manufacturera (INEGI, 2016); ii) producto interno bruto real manufacturero estatal a precios de 2008 (INEGI, 2016); iii) salarios reales (pesos por día), que corresponde al salario de cotización al Instituto Mexicano del Seguro 
Social (IMSS) por entidad federativa que publica la Secretaría del Trabajo y Previsión Social (STyPS, 2016); iv) exportaciones totales de México (INEGI, 2016); v) un indicador de productividad media del trabajo y; vi) inversión extranjera directa, que corresponde a los flujos de IED a México por entidad federativa de destino (SE, 2017).

Por la alta volatilidad de los flujos de la IED, se construyó una variable de acervo. En este caso se generó el stock de inversión extranjera directa a partir de los flujos anuales de IED para cada entidad federativa. La construcción de la variable de stock se realizó a partir del método de inventarios perpetuos (MIP), como lo sugieren Loría y De Jesús (2007), Shiau et al. (2002) y Almon (1999).

El MIP es un método relativamente sencillo que considera la tasa depreciación y acumula la inversión (en el caso que aquí se desarrolla, acumula el flujo de IED para expresarlo como una variable de stock). De manera formal se expresa de la siguiente forma:

$$
\text { kied }_{t}=(1-\mathrm{d}) * \text { kied }_{t-1}+\text { ied }_{t}
$$

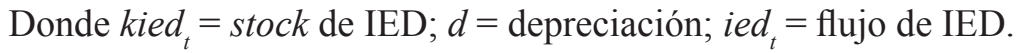

Siguiendo la recomendación de Shiau et al. (2002) y de Almon (1999), se incorpora el siguiente factor de ajuste: $A j_{t}=(1-\mathrm{d}) * A j_{t-1}+1$. Se supone que $A j_{t}=1$ para la observación inicial y crece hasta alcanzar el valor de equilibrio de la tasa de depreciación promedio igual a $1 / \mathrm{d}$. A partir de este factor de ajuste y de la estimación de kied por el MIP, se calcula una nueva

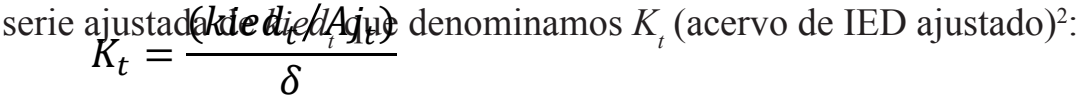

$K_{\mathrm{t}}$ es la variable de acervo de IED que se construyó para la estimación del panel. Se consideró una tasa de depreciación que varía entre 10 y 11 por ciento, ${ }^{3}$ que resulta del consumo de capital fijo trimestral que se reporta en el Sistema de Cuentas Nacionales de México (INEGI, 2016).

Se consideró un modelo con datos de panel por la estructura de la información estadística disponible para la industria manufacturera para las

\footnotetext{
${ }^{2}$ Para una revisión más detallada de las bondades y limitaciones de esta metodología, véase Loría y De Jesús (2007).

${ }_{3}^{3}$ Para la tasa de depreciación no existe consenso para definir la que corresponde a la economía mexicana, algunos autores suponen una tasa fija entre ocho por ciento (Blázquez y Santiso, 2004) y 12 por ciento (Shiau et al., 2002), otros, incluso, de cinco por ciento (Bergoeing et al. (2002).
} 
entidades federativas de México, y de esta forma agruparlas de acuerdo con las regiones de análisis. Además, porque los datos de panel permiten identificar y medir algunos efectos que no pueden considerarse al utilizar únicamente datos de corte transversal o datos de series temporales y porque permiten tener en cuenta la existencia de efectos individuales inobservables, que pueden estar correlacionados con otras variables incluidas en la especificación de una relación econométrica (véase Hsiao, 2003).

\section{La especificación del panel}

La especificación general de un modelo de regresión lineal con datos de panel es la siguiente (Hsiao, 2003):

$$
\mathrm{Y}_{\mathrm{it}}=\alpha+\beta \mathrm{X}_{\mathrm{it}}+\mathrm{u}_{\mathrm{it}}
$$

$\mathrm{i}=1, \ldots \mathrm{N}$;

$$
\mathrm{t}=1, \ldots \mathrm{T}
$$

Donde $i$ se refiere al individuo o a la unidad de estudio (corte transversal), $t$ a la dimensión en el tiempo, $\alpha$ es un escalar, $\beta$ es un vector de $K$ parámetros, $X_{\text {it }}$ es la i-ésima observación al momento $t$ para las $K$ variables explicativas y $u_{\mathrm{it}}$ es el término de error.

En este caso, la muestra total de las observaciones en el modelo vendría dada por $N$ x $T$, donde $N$ es el número de unidades de estudio individual y $T$ el periodo de tiempo.

Los modelos de panel se pueden interpretar a través de sus componentes de errores. El término de error $u_{\mathrm{it}}$ incluido en (1) puede descomponerse de la siguiente manera:

$$
\mathrm{u}_{\mathrm{it}}=\mu_{\mathrm{i}}+\delta_{\mathrm{t}}+\mathrm{e}_{\mathrm{it}}
$$

Donde $\mu_{\mathrm{i}}$ denota una variable no observable que se mantiene constante a través del tiempo para cada observación (efecto individual no observable), $\delta_{t}$ representa los efectos no cuantificables que varían en el tiempo pero no entre las unidades de estudio y $e_{\text {it }}$ se refiere al término de error. La mayoría de las aplicaciones con datos de panel utilizan el modelo de componente de error en una dirección: $\mathrm{u}_{\mathrm{it}}=\mu_{\mathrm{i}}+\delta_{\mathrm{t}}+\mathrm{e}_{\mathrm{it}}$, para el cual $\delta_{\mathrm{t}}=0$.

A partir de los distintos supuestos sobre los efectos específicos $\mu_{\mathrm{i}}$, pueden presentarse tres posibilidades: i) cuando se considera que $\mu_{\mathrm{i}}=0$, es decir, que no existe heterogeneidad no observable entre los individuos (por lo que se emplea la regresión agrupada); ii) cuando se supone a $m_{\mathrm{i}}$ como un efecto fijo y distinto para cada individuo, de modo que el modelo lineal es 
el mismo para todos los individuos pero la ordenada al origen es específica para cada uno de ellos. Consecuentemente, en este caso, la heterogeneidad no observable se incorpora a la constante del modelo y; iii) cuando se trata a $\mu_{\mathrm{i}}$ como una variable aleatoria no observable que varía entre individuos pero no en el tiempo. En este caso, las diferencias no observables se incorporan al término de error.

Estas variantes acerca de la heterogeneidad no observable dan lugar a dos tipos de modelos: el modelo de efectos fijos y el modelo de efectos aleatorios (Montero, 2007; Wooldridge, 2002).

El modelo de efectos fijos

$\mathrm{Y}_{\mathrm{it}}=\alpha+\mathrm{X}^{\prime}{ }_{\mathrm{it}} \beta+\mu_{\mathrm{i}}+\mathrm{e}_{\mathrm{it}}$

$\mathrm{O}$ bien

$\mathrm{Y}_{\mathrm{it}}=\alpha+\mathrm{X}_{\mathrm{it}} \beta+\mathrm{e}_{\mathrm{it}} ; \alpha_{\mathrm{i}}=\alpha+\mu_{\mathrm{i}}$

Supone que el error $u_{\mathrm{it}}$ expresado en (1) puede descomponerse en una parte puramente aleatoria y otra fija constante y diferente para cada individuo $\mu_{\mathrm{i}}$ (considerada como un parámetro a estimar para cada observación), lo cual es equivalente a realizar una regresión general y dar a cada individuo un punto de origen (ordenadas) distinto, incorporando así la heterogeneidad a la constante del modelo $\alpha_{\mathrm{i}}=\alpha+\mu_{\mathrm{i}}$ De esta forma, en el modelo de efectos fijos los son tratados como un conjunto de $n$ coeficientes adicionales que se pueden estimar junto con los $\beta_{\mathrm{s}}$. Asimismo, el modelo de efectos fijos asume que el efecto individual está correlacionado con los demás regresores, es decir, $\operatorname{cov}\left(X_{\mathrm{it}}, \mu_{\mathrm{i}}\right) \neq 0$ (Montero, 2007; Wooldridge, 2002).

El modelo de efectos aleatorios

Yit $=\alpha+X^{\prime}{ }_{\text {it }} \beta+\mu_{\mathrm{i}}+\mathrm{e}_{\mathrm{it}}$

$\mathrm{O}$ bien

$$
\mathrm{Y}_{\mathrm{it}}=\alpha+\mathrm{X}_{\mathrm{it}} \beta+\mathrm{u}_{\mathrm{it}}, \mathrm{u}_{\mathrm{it}}=\mu_{\mathrm{i}}+\mathrm{e}_{\mathrm{it}}
$$

Tiene la misma especificación que el de efectos fijos sólo que $\mu_{\mathrm{i}}$, en lugar de ser un valor fijo para cada individuo y constante a lo largo del tiempo, es una variable aleatoria. Como en el modelo de efectos aleatorios se supone que $\mu_{\mathrm{i}}$ es una variable aleatoria inobservable independiente de $X_{\mathrm{it}}$, ésta pasa a formar parte de un término de perturbación compuesto 
$\mu_{\mathrm{i}}=\mu_{\mathrm{i}}+\mathrm{e}_{\mathrm{it}}$; incorporando así la heterogeneidad no observable al término de error en lugar de la constante como en el caso de efectos fijos (Arellano y Bover, 1990).

Así, a partir de la ecuación (1) se puede estimar la función de empleo manufacturero para cada una de las regiones de México en términos de un modelo general de regresión lineal con datos de panel.

De esta forma, se define una función de empleo en los siguientes términos:

tcman $_{\text {it }}=\alpha_{i}+\alpha_{1}$ tcpibm $_{\text {it }}+$ tckied $_{\text {it }}+$ tcexptot $_{\text {it }}+$ tcwreal $_{\text {it }}+$ tcprodlab $_{\text {it }}+v_{\text {it }}$

Donde $t c m a n_{\text {it }}$ es la tasa de crecimiento del empleo manufacturero por entidad federativa para cada región; tcpibm $_{\text {it }}$ es la tasa de crecimiento del producto interno bruto real manufacturero, por entidad federativa para cada región; tkied $_{\mathrm{it}}$ es la tasa de crecimiento del stock de IED (generado a partir del MIP referido anteriormente) por entidad federativa para cada región; tcexptot $_{\text {it }}$ son las exportaciones totales del sector manufacturero de México en tasas de crecimiento, tcwreal $_{\mathrm{it}}$ es la tasa de crecimiento de los salarios reales (pesos por día), que corresponde al salario de cotización al IMSS por entidad federativa; tcprodla $_{\text {it }}$ la tasa de crecimiento de la productividad media del trabajo manufacturero por entidad federativa para cada región. Finalmente, $v_{\text {it }}$ son los errores.

De esta función de empleo se asumen las siguientes relaciones entre la tasa de crecimiento del empleo, como variable dependiente, y las variables que se han considerado como determinantes o independientes: positiva con la producción (tcpibm), en el sentido de que la actividad manufacturera de cada entidad federativa es central en la generación de empleo, desde una visión estrictamente keynesiana de demanda efectiva en el sentido de que cuando una empresa satisface la demanda de su producto, emplea exactamente la cantidad de trabajo necesaria para producir la cantidad demandada, es decir, las empresas deben emplear más trabajo cuando la demanda de producción es más alta (Abel y Bernanke, 2004); positiva con la inversión extrajera directa, que en este caso es el stock de IED (tckied); positiva con la dinámica de las exportaciones manufactureras (tcexptot), bajo el supuesto de que el proceso de apertura comercial ha favorecido el incremento de las exportaciones, de la producción y, en consecuencia, del empleo; negativa con los salarios (tcwreal), desde un argumento neoclásico en el sentido de que los incrementos del costo de la mano de obra por incremento en salarios desestimulan la contratación laboral y, por lo tanto, los niveles 
de empleo; negativa con el producto medio del trabajo (tcprodlab) por un efecto de desplazamiento del trabajo por incrementos de productividad.

\section{Estimación y discusión de resultados}

Se integraron cinco paneles, uno para cada una de las regiones definidas en el apartado 2. Son paneles balanceados con cortes transversales de ocho periodos que corresponden a la información anual del periodo 2007-2014.

Se estimó la función (4) para el periodo 2007-2014, para el total de la economía y para cada una de las cinco regiones de México. Primero, la función de empleo para el total de la manufactura del país con el propósito tener un panorama global que proporcionará información de la estructura del empleo en general para, posteriormente, evaluar si con la misma función de demanda de empleo se podrían identificar regularidades similares para las regiones de México; sobre todo, que permitiera evaluar el peso que la IED tiene en la generación de empleo manufacturero para el total de la economía mexicana y en las diferentes regiones.

A partir de la metodología convencional, se estimó el panel con datos agrupados (pool), con efectos fijos y con efectos aleatorios; posteriormente se compararon los resultados de los modelos de efectos individuales (fijos $\mathrm{y}$ aleatorios) con relación al modelo de datos agrupados; y a partir de las pruebas de datos agrupados y de Hausman se evaluó el mejor modelo. En este sentido, primero se comparó el modelo de efectos fijos con el modelo de datos agrupados para evaluar la eficiencia del modelo de efectos fijos. Para ello, la prueba de datos agrupados utiliza una prueba de restricción de parámetros entre los dos modelos y se analizan las hipótesis: $H_{0}$ : "para todo $\mu_{\mathrm{i}}=0$ y $H_{1}: \mu_{1} \neq 0, \ldots, \mu_{\mathrm{i}} \neq 0$. Si la hipótesis nula $\mathrm{H}_{0}$ no se puede rechazar, se opta por el modelo de datos agrupados, en caso contrario se elige el de efectos fijos.

De las pruebas de datos agrupados y de Hausman (véase resultados de las pruebas en la Tabla 2), se concluyó que para el total de la manufactura de México y para las regiones el modelo de efectos fijos es el más consistente.

Entre los principales resultados, destacan los siguientes:

- Para el empleo total de la manufactura nacional se reportan resultados consistentes, de acuerdo con la magnitud y signos de los coeficientes estimados, además de que son estadísticamente significativos; a excepción de la inversión extranjera directa (tckied), cuyo coeficiente, aunque positivo de 0.001 , resultó no significativo. De manera específica, 
se registra un coeficiente de 0.550 para tcpibm; que estaría indicando que el crecimiento del empleo manufacturero se explica de manera importante por el crecimiento de la producción manufacturera (por cada uno por ciento que aumente la tasa de crecimiento del PIB de la manufactura el crecimiento del empleo aumentará en 0.550 por ciento); en el caso de la IED se podría argumentar que para los años de estudio (2007-2014) los efectos que el crecimiento del stock de capital generado por los flujos de IED tiene en el crecimiento del empleo es mínimo (coeficiente de tckied de 0.001), lo que permitiría argumentar que la IED no ha tenido el impacto esperado, al menos en la generación de empleo. Los resultados dejan ver que después del PIB de la manufactura hay un mayor peso relativo en los determinantes del empleo de los salarios reales, la productividad del trabajo y, finalmente, de las exportaciones, que de la IED.

- El signo positivo de los salarios (tcwreal) estaría sugiriendo que aumentos en el empleo se asocian a aumentos en los salarios, lo que llevaría a argumentar que por la estructura de producción de la manufactura deberá analizarse a partir de la teoría de los salarios de eficiencia, como lo sugieren Carbajal y de Jesús (2017). Recuérdese que los salarios de eficiencia son las cantidades que las empresas pagarían por encima del valor del salario de equilibrio del mercado para evitar caídas en la productividad (Malcomson, 1981 y Gordon, 1990).

- Finalmente, el coeficiente de la productividad (tcprodlab) (negativo y estadísticamente significativo) aporta evidencia de un efecto de desplazamiento de trabajo por productividad que, aunque marginal (coeficiente de -0.361), se puede asociar al tipo de inversión que llega a la manufactura y que se relaciona con inversión acompañada de innovación tecnológica.

Estos resultados sugieren evaluar lo que ocurre con las regiones, bajo el argumento de que el proceso global de apertura y de captación de flujos de IED no tiene los mismos efectos en todas las regiones, sobre todo porque se sostiene la hipótesis de que aquellas regiones más vinculadas al sector externo vía comercio son las más beneficiadas en la captación de IED y, en consecuencia, tenderían a ser las que mejores efectos tendrían en el crecimiento de la producción y en la generación de empleos.

Para las regiones es clara la heterogeneidad en la estructura de los factores que explican el crecimiento del empleo manufacturero. Los resultados de la estimación (Tabla 2) reportan, primero, un coeficiente positivo de tcpibm para todas las regiones, lo que validaría la relación positiva que se 
supone existe entre crecimiento del PIB manufacturero y crecimiento del empleo; sin embargo, la magnitud del coeficiente del crecimiento del PIB de la manufactura para las regiones es diferente: la región Norte registra el más alto (0.898), la Frontera Norte un coeficiente de 0.443, le siguen la Centro Este y Centro Oeste con estructuras semejantes (0.368 y 0.346 , respectivamente) y, finalmente, la región Sur (0.257).

Se deben destacar los resultados del coeficiente de estimación de la inversión extranjera directa (tckied). Como se observa, no hay evidencia de que en las regiones la acumulación de IED sea un factor que influya en la generación de empleo manufacturero (además de que los coeficientes de estimación son pequeños, no son significativos); sin embargo, habría que cuestionar si las magnitudes del coeficiente para las regiones corresponden a los efectos que se esperaría debería tener la IED en el empleo. Más aún, estos resultados nos proporcionan elementos para considerar la necesidad de abundar en el análisis regional a un nivel más detallado para mejorar las estimaciones y llegar a resultados que abunden en las posibles virtudes que la IED tiene en las economías regionales.

Como se sabe, la manufactura es de los sectores más vinculados al sector externo, de ahí que se considere que las exportaciones del sector (tcexptot) son uno de los principales determinantes del empleo. Los coeficientes de estimación para tcexptot son positivos en todas las regiones; sin embargo, para las regiones Norte y Centro Oeste no son significativos. Más aún, la Frontera Norte y la Centro Este registran un coeficiente mayor que la estimación para el total del empleo manufacturero de México (0.188 y 0.155 , para la Frontera Norte y Centro Este, respectivamente, mientras para el total nacional es de 0.053 ).

Finalmente, los resultados para los salarios y la productividad reportan lo siguiente: no hay evidencia suficiente de que en las regiones el crecimiento de los salarios reales sean un factor que explique el crecimiento del empleo manufacturero, en el sentido de que aumentos en los salarios reducen la creación de empleo porque se asocian a incrementos en los costos de producción. En el caso de la productividad del trabajo, la evidencia para las regiones parece consistente con la estructura que prevalece para el total del empleo manufacturero en México (el coeficiente de tcprodlab para el total del empleo manufacturero es de -0.361); a excepción de la re- 
Inversión extranjera directa y empleo manufacturero. Un análisis regional con datos ... / L. de J. ALMONTE et al.

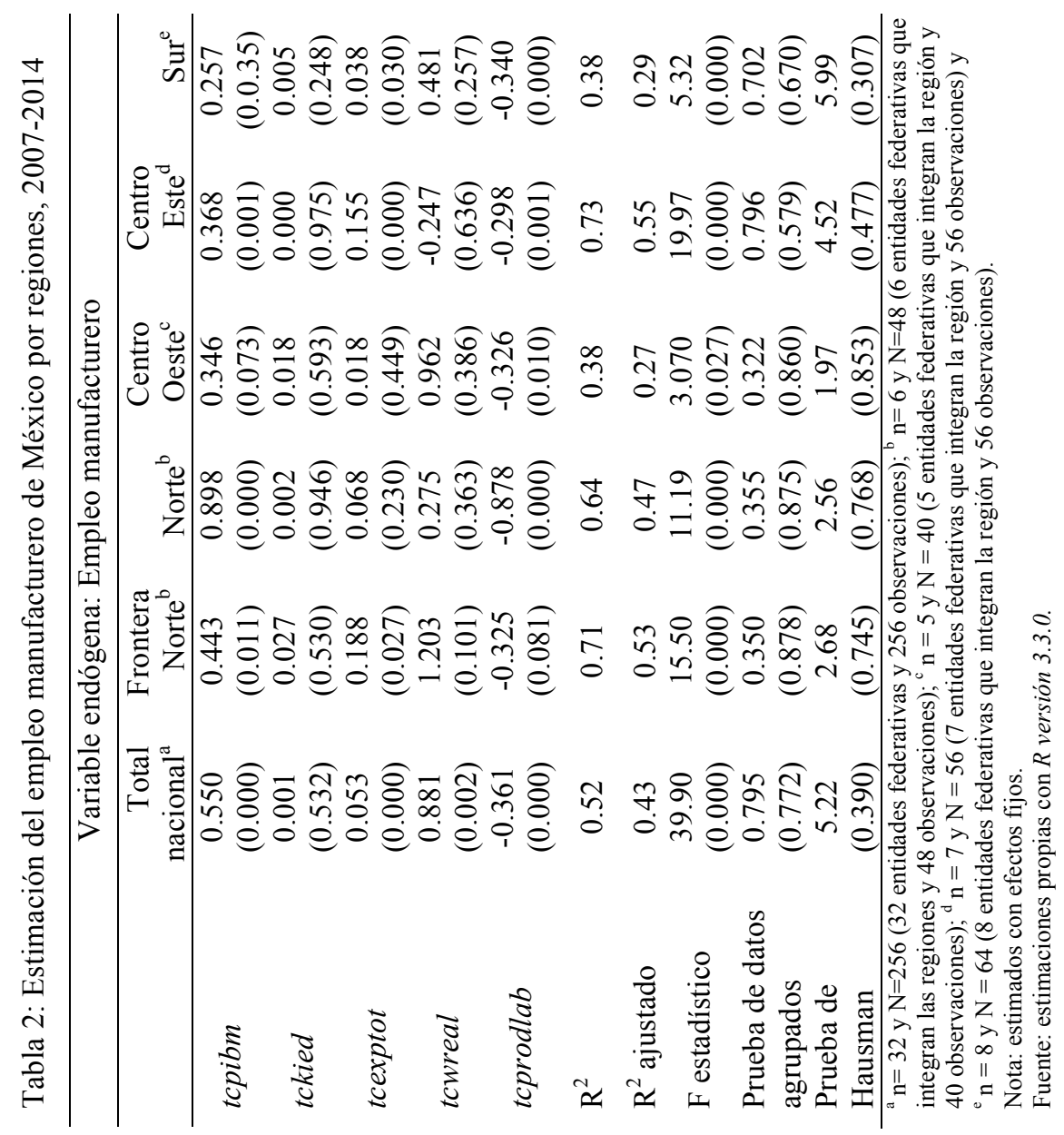


gión Norte, cuyo coeficiente es el más alto (-0.878), y de la región Centro Este, con el coeficiente más bajo (-0.298), las demás regiones registran un coeficiente similar.

Este hecho es importante porque es aceptado que parte importante de los flujos de IED que se destinan a la producción van acompañadas de innovación tecnológica, de ahí que se pueda argumentar que la productividad laboral (tcprodlab) se está convirtiendo en un factor que desplaza trabajo, de acuerdo con lo que reportan el signo negativo de los coeficientes para el total de la economía mexicana y para las regiones (Tabla 2).

\section{Conclusiones}

La relación entre IED y la generación de empleos en México ha sido una línea de investigación poco analizada desde la literatura del trabajo académico. Este documento incursiona en esta línea de análisis y aporta elementos que permitan entender los efectos de esta inversión a nivel regional en cuanto a la generación de empleos vinculados a la manufactura.

La dinámica de los flujos de inversión en México se configuró a raíz de la apertura de la economía y, particularmente, por la incorporación al Tratado de Libre Comercio con América del Norte. La integración comercial con Estados Unidos determinó el sesgo exportador-maquilador que adquirió la IED y explica la relevancia del sector manufacturero como destino de los capitales extranjeros.

No obstante, con la finalidad de observar las heterogeneidades de la economía mexicana, el análisis de la IED se realizó a nivel regional. Más allá del sesgo que presentó la región Centro Oeste en la concentración de los flujos de IED, por efectos de domiciliación fiscal de las empresas, las regiones Frontera Norte, Norte y Centro Este presentaron trayectorias interesantes en cuanto a la captación de los flujos de inversión que se asociaron a sus propias condiciones socioeconómicas y que les permitieron sobrepasar la Gran Recesión. Entidades federativas como Durango, Zacatecas o Guanajuato prácticamente duplicaron la captación de IED en el periodo posterior a 2008 .

La evidencia empírica, a partir de las estimaciones realizadas, demuestra que existe una relación muy importante entre el crecimiento del PIB y el crecimiento del empleo de la manufactura para la estimación del total del empleo manufacturero de México y para cada una de las regiones. El coeficiente del crecimiento del PIB de la manufactura de 0.550 , indica que el crecimiento del empleo manufacturero se explica de manera importante por el crecimiento de la producción manufacturera; que para las regiones 
se estima entre 0.898 , que es el más alto y que corresponde a la región Norte, y 0.257 , el más bajo que corresponde a la región Sur.

Con relación al efecto que la IED tiene en la generación de empleos, nuestros resultados coinciden con estudios similares en cuanto a la metodología empleada para la estimación del IED en el empleo en los países de Europa Central y Oriental (Jude y Pop, 2015; Onaran, 2008). Sin embargo, no se encontró evidencia consistente de que en las regiones la acumulación de IED sea un factor que influya en la generación de empleo manufacturero, los coeficientes de estimación son pequeños y no son significativos, lo que proporciona elementos para concluir que la IED no ha tenido los efectos esperados, al menos en la generación de empleo, sobre todo porque los resultados dejan ver que, después del PIB de la manufactura, hay un mayor peso relativo en los coeficientes estimados de la productividad laboral y de las exportaciones para algunas regiones, específicamente para la Centro Este.

Por último, los resultados del estudio nos proporcionan elementos para considerar la necesidad de continuar con esta línea de análisis regional a un nivel más detallado para mejorar las estimaciones y tener resultados que abunden en las posibles virtudes que la IED tiene en las economía regionales que, como se observa, son efectos heterogéneos.

\section{REFERENCIAS BIBLIOGRÁFICAS}

Abel, A. B. y Bernanke, B. S., 2004, Macroeconomía. España: Pearson-Addison Wesley.

Aguayo Téllez, Ernesto, 2004, “Divergencia Regional en México, 1990-2000”, en Ensayos: Revista de economía, 23 (2). pp. 30-42, disponible en www.economia. uanl.mx/revistaensayos/xxiii/2/Divergencia_regional.pdf

Aitken, B., J., and Harrison, A. E., 1999, "Do domestic firms benefit from direct foreign investment? Evidence from Venezuela", in American Economic Review, vol. 89 (3), pp. 605-18, disponible en http://pubs.aeaweb.org/doi/pdfplus/10.1257/ aer.89.3.605 (30/02/17).

Almon, C., 1999, The Craft of Economic Modeling, Needham Heights. MA: Ginn Press.

Ángeles-Castro, Gerardo y Ortiz-Galindo, Jonathan, 2010, "La inversión extranjera directa en México y su efecto en el crecimiento y la desigualdad económica", en Eseconomía, vol. 5, 26, pp. 95-123, disponible en http://yuss.me/revistas/ese/ ese2010v05n26a05p095_123.pdf 
Arellano, M. y Bover, O., 1990, "La econometría de datos de panel”, en Investigaciones Económicas, 14(1): 3-45, disponible en ftp://ftp.fundacionsepi.es/InvEcon/ paperArchive/Ene1990/v14i1a1.pdf

Banco de México, 2016, Sistema de Información Económica, disponible en http:// www.banxico.org. $\mathrm{mx} /$ SieInternet/consultarDirectorioInternetAction.do?sector=1 \&accion $=$ consultarCuadro\&idCuadro $=\mathrm{CE} 146 \&$ locale $=\mathrm{es}$

Banco Mundial, 2016, Datos, disponible en http://datos.bancomundial.org/indicador/

Bergoeing, R.; Kehoe, P. y Soto, R., 2002, “A Decade Lost and Found: Mexico and chile in the 1980s", in Review of Economic Dynamics, 5:166-205, disponible en http://www.sciencedirect.com/science/article/pii/S1094202501901504

Blázquez, J. y Santiso, J., 2004, “Mexico: Is it an ExEmerging Market”, in Journal of Latin American Studies, 36:297-318.

Carbajal Suárez, Y. y Almonte, L. de Jesús, 2017, "Manufacturing labor in the Central Region of Mexico. An estimation by great division, 1985-2008", en Contaduría y Administración, http://dx.doi.org/10.1016/j.cya.2017.05.004. En prensa.

Carbajal Suárez, Y.; Almonte, L. de Jesús y Mejía Reyes, P., 2016, "La manufactura y la industria automotriz en cuatro regiones de México. Un análisis de su dinámica de crecimiento, 1980-2014", en Economía: Teoría y Práctica. Nueva Época, número 45, julio-diciembre 2016, pp. 39-66, disponible en http://www.izt. uam.mx/economiatyp/ojs

CEPAL, 2013, La inversión extranjera directa en América Latina y el Caribe 2012, Comisión Económica de América Latina y El Caribe. Santiago de Chile.

CEPAL, 2016, La inversión extranjera directa en América Latina y el Caribe 2012, Comisión Económica de América Latina y El Caribe. Santiago de Chile.

Chiatchoua, Cesaire; Neme Castillo, Omar y Valderrama Santibáñez, Ana Lilia, 2016, "Inversión Extranjera Directa y empleo en México: análisis sectorial", en Economía Informa. 398. Facultad de Economía, UNAM. Pp. 40-59, disponible en http://www.economia.unam.mx/assets/pdfs/econinfo/398/03chictchoua.pdf

Díaz Carreño, M. Á.; Morales Fajardo, M. E. y Vergara González, R., 2011, “Comercio exterior, inversión extranjera directa y actividad económica de México, 1970-2008”, en Mejía Reyes, P. y Morales Fajardo, M. E. (coords.), Integración y recesión económica en el binomio México-Estados Unidos. Universidad Autónoma del Estado de México. Toluca.

Dussel Peters. E.; Galindo, L. M. y Loría, E. (coords.), 2003, Condiciones y efectos de la Inversión Extranjera Directa y del proceso de integración regional en México durante los noventa: Una perspectiva macro, meso y micro, UNAM, Banco Interamericano de Desarrollo y Plaza y Valdes. México.

González López, S. y Hernández Medina, M. L., 2008, "Las transformaciones económicas regionales: la inversión extranjera directa entre lo local y lo global", en Mejía Reyes, P.; del Moral, L. E. y Rodríguez, O. M. (coords.), Actividad eco- 
nómica en el Estado de México. Vol. I. Desempeño productivo y sector externo. Biblioteca Mexiquense del Bicentenario. Gobierno del Estado de México.

Gordon, R., 1990, What is new keynesian economics?, in Journal of Economic Literature, 28 (3), 1115-1171, disponible en http://public.econ.duke.edu/ kdh9/ Courses/Graduate por ciento20Macro\%20History/Readings-2/Gordon_Keynesian\%20Economics.pdf

Gouëset, Vincent, 2000, “El impacto territorial de la inversión extranjera en América Latina. Estudio comparativo de tres casos en México, Argentina y Colombia", en Territorios, consulta: 13/02/2017, Disponible en http://222.redalyc.org/articulo.oa? id=35700404, ISSN 0123-8418

Hanousek, J; Kocenda, E. y Maurel, M., 2011, "Direct and indirect effects of FDI in emerging European markets: a survey and meta-analysis", in Economis Systems, vol. 35 (3), pp. 301-322, http://dx.doi.org/10.1016/j.ecosys.2010.11.006

Hsiao, Ch., 2003, Analysis of Panel Data. Cambridge Books, Cambridge University Press.

INEGI, 2016, Banco de Información Económica. Instituto Nacional de Estadística y Geografía. México, disponible en http://www.inegi.org.mx/sistemas/bie/

Jude, C. y Pop S., 2015, "Employment effects of foreign direct investment: New evidence from Central and Eastern European countries", in International Economics, vol. 145, (mayo), pp. 32-49. http://dx.doi.org/10.1016/j.inteco.2015.02.003

Loría Díaz, E. y Brito Cruz, L., 2005, "El impacto de la inversión extranjera directa en el empleo sectorial en México: un análisis prospectivo", en Análisis Económico. XX (segundo cuatrimestre): consulta: 30/08/2016, Disponible en http:// www.redalyc.org/articulo.oa? $\mathrm{id}=41304402$

Loría Díaz, E. y L. de Jesús Almonte, 2007, “Los acervos de capital de México. Una estimación, 1980.I-2004.IV”, en El Trimestre Económico. 74(294):475-485.

Malcomson, J., 1981, "Unemployment and the efficiency wage hypothesis", in The Economic Journal, 91 (364), 848-866.

Mejía R. Pablo; Hurtado, Annel y Vergara, Reyna, 2013, “QQué explica la inversión privada en México?, en Torres, Víctor H.; Tinoco, Miguel y Polanco, Mayrén (coords.), Los desafios de la economía mexicana. Inversión y crecimiento económico. pp. 17-52.

Mejía, Pablo, 2005, "La inversión extranjera directa en los estados de México: Evolución reciente y retos futuros", en Economía, Sociedad y Territorio, Número especial, 185-237. El Colegio Mexiquense. Toluca. http://132.248.9.34/hevila/ Economiasociedadyterritorio/2004-05/vol4-5/noesp/6.pdf

Mendoza Cota, Jorge Eduardo, 2011, "Impacto de la inversión extranjera directa en el crecimiento manufacturero en México", en Revista Problemas del Desarrollo. 167 (42). UNAM, México, pp. 45-69, disponible en http://www.revistas.unam. $\mathrm{mx} /$ index.php/pde/article/view/27720 
Montero, R., 2007, Efectos fijos o variables: test de especificación, Documento de trabajo, Universidad de Granada, España.

Morales Fajardo, M. E.; Almonte L. de Jesús y Ávila Morales, M. E., 2015, “Inversión extranjera directa en América Latina: una revisión al panorama regional en la primera década del siglo XXI", en Morales Fajardo, M. E. y Almonte, L. de Jesús (coordinadores), Inversión Extranjera Directa en América Latina. Una revisión en las economías latinoamericanas en los albores del siglo XXI. UNAM, México.

Onaran, O., 2008, "Jobless growth in the Central and East European countries: a country-specific panel data analysis of the manufacturing industry", in Eastern European Economics, vol. 46, (4), (Jul-Aug), pp. 90-115. http://www.jstor.org/ stable/27740077 (02/02717).

Romero Tellaeche, José Antonio, 2012, "Inversión extranjera directa y crecimiento económico en México, 1940-2011", en Investigación Económica. 71 (282): 109-147. Disponible en http://www.scielo.org.mx/scielo.php?script=sci arttext\&pid=S0185-16672012000400005\&lng=es\&tlng=es. Recuperado en 30/08/2016.

Romero Tellaeche, José Antonio, 2014, Los límites al crecimiento económico de México. El Colegio de México-UNAM. México.

Secretaría de Economía, 2016, Reportes estadísticos de IED. Disponible en http:// www.gob.mx/se/acciones-y-programas/competitividad-y-normatividad-inversion-extranjera-directa?state $=$ published

Secretaría de Economía, 2017, Información estadística de flujos de IED hacia.... disponible en https://datos.gob.mx/busca/dataset/inversion-extranjera-directa/ resource/083cc955-6474-4441-a238-b81e15ca82de (13 de enero de 2017)

Shiau, A.; Kilpatrick, J. y Matthews, M., 2002, "Seven Percent Growth for Mexico? A Quantitative Assessment of Mexico's Investment Requirements", Journal of Policy Modeling. 24(7-8):781-798.

STyPS, 2016, Estadísticas del Sector. Secretaría del Trabajo y Previsión Social, México. Disponible en http://www.stps.gob.mx/gobmx/estadisticas/

Torres Preciado, V.; Polanco Gaytán, M. y Tinoco Zermeño, M., 2017, "Dinámica de la inversión extranjera directa en los estados de México: un análisis de cadenas de Markov espaciales", en Contaduría y Administración, 62(1), 141-162. Recuperado de http://www.cya.unam.mx/index.php/cya/article/view/867

Trevino, Len J.; Daniels, John D. and Arbeláez, Harvey, 2002, "Market reform and FDI in Latin America: an empirical investigation", in Transnational Corporations, vol. 11, No. 1. UNCTAD. Pp. 29-48. Disponible en http://unctad.org/en/ docs/iteiit29v11n1a2_en.pdf, Revisado (30/08/2016).

Turner Barragán, E. H. y Martínez Pérez, J. F., 2003, "Inversión extranjera y empleo en México", en Análisis Económico, 18 (primer semestre): Fecha de consulta: 30 de agosto de 2016, disponible en http://www.redalyc.org/articulo. oa? $\mathrm{id}=41303711>$ ISSN $0185-3937$ 
UNCTAD, 2016, The World Investment Report 2016. Investor Nationality: Policy Challenges. United Nations Conference on Trade and Development. http://unctad. org/en/PublicationsLibrary/wir2016_en.pdf

Vergara, R. y Carbajal, Y., 2015, “La inversión extranjera directa en México, 19942012. Un análisis de autocorrelación espacial”, en Morales, Ma. E. y Almonte, L. de Jesús (coords.), Inversión Extranjera Directa en América Latina. Una revisión de las economías latinoamericanas en los albores del siglo XXI, UNAM-CRIM, México.

Vergara González, R.; Almonte, L. de Jesús y Carbajal Suárez, Y., 2015, “IED y empleo en la región norte de México, 2004.1-2013.4. Un análisis para el sector industrial", en Equilibrio Económico, vol. 11(1), núm. 39, disponible en http://www. equilibrioeconomico.uadec.mx/descargas/Rev2015/Rev15Sem1Art3.pdf

Wooldridge, J., 2002, Econometric Analysis of cross section and panel data. The MIT Press, Cambridge, Massachusetts, London, England.

\section{RESUMEN CURRICULAR DE LOS AUTORES}

\section{Leobardo de Jesús Almonte}

Doctor en Economía por la Universidad Nacional Autónoma de México. Actualmente es profesor investigador de tiempo completo del Centro de Investigación en Ciencias Económicas de la Facultad de Economía de la Universidad Autónoma del Estado de México y director editorial de Paradigma Económico, revista de economía regional y sectorial de la UAEM. Perfil deseable Prodep. Entre sus publicaciones recientes destacan: Empleo manufacturero en la Región Centro de México. Una estimación por gran división (en coautoría con Yolanda Carbajal Suárez), Empleo en el sector terciario. Una estimación espacial para los municipios de la región centro de México, 1999-2009 (en coautoría con Yolanda Carbajal Suárez), Inversión fija bruta y empleo manufacturero en México, 2008 y 2012. Un análisis de insumo-producto (en coautoría con Brenda Murillo Villanueva).

Dirección electrónica:1dejesusa@uaemex.mx

\section{Maria Esther Morales Fajardo}

Doctora en Ciencias Sociales con especialidad en Relaciones Internacionales por la Universidad Autónoma Metropolitana-Xochimilco. Es investigadora titular del Centro Regional de Investigaciones Multidisciplinarias de la Universidad Nacional Autónoma de México y profesora de asignatura de la Facultad de Economía de la Universidad Autónoma del Estado de México. Es integrante del Sistema Nacional de Investigadores. Entre sus publicaciones se encuentran: Engagement to Global Production Networks 
in Southeast Asia: prospects for technology upgrade and lessons for Latin America (en coautoría con J.F. López Aymes) e Inversión Extranjera Directa en América Latina: una revisión en los albores del siglo XXI (en coordinación con L. de Jesús Almonte).

Dirección electrónica: moralesf@correo.crim.unam.mx

Yolanda Carbajal Suárez

Doctora en Economía por la Universidad Nacional Autónoma de México. Pertenece al Sistema Nacional de Investigadores Nivel 1. Profesorainvestigadora de tiempo completo definitiva de la Facultad de Economía de la Universidad Autónoma del Estado de México. Miembro del Centro de Investigación en Ciencias Económicas. Coordinadora del Observatorio Estatal del Estado de México. Sus líneas de investigación son: Economía regional y sectorial y sector automotriz. Entre sus publicaciones recientes se encuentran: Dinámica productiva del sector automotriz y la manufactura en la frontera norte de México: Un análisis con datos de panel, 19802014 (en coautoría con Leobardo de Jesús y Berenice Carrillo); Empleo en el sector terciario. Una estimación espacial para los municipios de la región Centro de México, 1999-2009”, (en coautoría con Leobardo de Jesús); La manufactura y la industria automotriz en cuatro regiones de México. Un análisis de su dinámica de crecimiento, 1980-2014, (en coautoría con Leobardo de Jesús y Pablo Mejía); Evolución, condiciones actuales y retos del sector automotriz en México y en el Estado de México.

Dirección electrónica: ycarbajals@uaemex.mx

Artículo recibido el 1 de junio de 2017 y aprobado el 6 de junio de 2018. 